INRA Prod. Anim., 2004, 17 (3), 211-215

\section{M.-L. BEGOUT ANRAS, J.P. LAGARDERE}

Centre de Recherche sur les Ecosystèmes

Marins et Aquacoles, UMR 10

CNRS/IFREMER, BP 5,

F-17137 L'Houmeau

Courriel :

Marie.Laure.Begout@ifremer.fr

\title{
Domestication et comportement chez les poissons téléostéens
}

\begin{abstract}
Les recherches sur les animaux domestiques fournissent de nombreux exemples de transformation des comportements. Avec la protection des murs, des enclos, des stalles, l'activité sensorielle et les aptitudes physiques des animaux domestiques ont souvent beaucoup moins d'importance qu'un taux élevé de croissance ou de reproduction.
\end{abstract}

Il en est probablement de même pour les poissons, cependant les recherches portant directement sur leur domestication, processus d'adaptation des organismes à un environnement procuré par l'homme, sont peu nombreuses (revue : Ruzzante 1994). Néanmoins, diverses études réalisées sur l'aptitude des poissons, issus d'élevage en vue de repeuplement, à retourner à la vie sauvage nous révèlent peu à peu les types de comportements modifiés par le maintien plus ou moins prolongé en élevage. Il est indiscutable

\section{Résumé}

Un des principaux objectifs de la domestication est de sélectionner des lignées à haut potentiel de croissance et à faible agressivité. L'étude des caractéristiques des animaux domestiqués montre souvent de nombreux changements comportementaux dus aux conditions d'élevage, mais très peu de données sont disponibles chez les poissons. Cet article décrit d'abord comment la mise en élevage affecte certains comportements, notamment alimentaires et natatoires, puis présente les modifications, au cours du développement, des organes sensoriels et des aptitudes comportementales. Concernant le comportement alimentaire, les modifications sont principalement dues au fait que la distribution d'aliment ne correspond pas nécessairement au rythme biologique des poissons et que la quantité d'aliment disponible est souvent bien supérieure en élevage comparé au milieu naturel. Le mode de distribution peut également influencer l'agressivité. Les conditions d'élevage (courant, densité animale ...) peuvent avoir des conséquences sur l'agressivité des poissons, en modifiant leur répartition spatiale, avec en particulier une réduction de la distance interindividuelle.

Le développement des poissons en milieu d'élevage semble affecter certaines aptitudes comportementales en relation avec des modifications de leurs organes sensoriels (yeux, ligne latérale et odorat). Ces modifications sont généralement associées à une diminution de la vigilance avec notamment des conséquences sur la capacité à échapper aux prédateurs potentiels. Chez les poissons plats, le maintien en élevage modifie également l'adaptation chromatique.

En conclusion, l'analyse des modifications comportementales induites par la domestication est un outil nécessaire pour identifier les facteurs à prendre en compte afin d'identifier la mise en place du processus de domestication, de garantir le bien-être des poissons et ainsi la durabilité du système d'élevage. que les traits comportementaux sont parmi les premières caractéristiques d'une espèce (mammifère ou poisson) à être affectés par le processus de domestication (Mayr 1963, Kohane et Parson 1988, Ruzzante 1994) et, habituellement, c'est la fréquence ou l'intensité avec laquelle un comportement particulier s'exprime qui est affecté par la domestication, plutôt que le type de comportement lui-même (Hale 1969, Boice 1972, Price 1984, Ruzzante 1994). Par ailleurs, il est assez généralement admis que ces changements de fréquence ou d'intensité dans un comportement particulier sont davantage liés à des déplacements des seuils de sensibilité qu'à des modifications génétiques. Ceci fait dire à Tsukamoto et al (1999) que plus la domestication avance, plus la qualité comportementale décroît.

Partant de ces constatations de base, notre premier objectif va être de rechercher les principaux types de comportement affectés par un maintien plus ou moins prolongé en élevage. Le second sera d'attirer l'attention sur les organes sensoriels et les capacités locomotrices susceptibles d'être modifiés par la vie en milieu artificiel. Les aspects génétique et adaptation chez les poissons ne seront pas traités ici car ils ont fait l'objet d'un article récent (Vandeputte et Prunet 2002). Enfin, nous essayerons de dégager, parmi toutes ces modifications, celles qui sont susceptibles de servir d'indicateurs pour une évaluation pratique de la progression du processus de domestication.

\section{1 / Comportements affectés par le maintien en élevage}

L'expression d'un comportement apparaît comme dépendante de la force des stimuli déclencheurs et de la disposition endogène (état physiologique) de l'animal (EiblEibesfeldt 1972). Par ailleurs, l'ensemble des comportements ou options comportementales s'ordonne en une organisation hiérarchique au sein de laquelle le choix d'une de ces options ou d'une succession d'entre elles représente une tactique. A long terme, le succès d'une tactique dépend de sa pertinence et de la fréquence de sélection qui lui est asso- 
ciée dans le contexte d'une stratégie particulière qui évolue ainsi vers une solution stable. Un exemple de stratégie peut être celui des migrations opérées de façon récurrente par les poissons amphihalins.

Les facteurs primordiaux, stimuli, qui vont diriger les réponses comportementales d'un poisson dans un environnement naturel sont liés à son environnement physique, à la disponibilité en proies et à leur accessibilité, au comportement des autres poissons, aux risques de prédation et, enfin, à l'état interne de l'individu. Lorsque l'on considère le contexte aquacole, l'environnement physique et l'accessibilité à l'aliment inerte restent des facteurs importants qui vont principalement affecter l'agressivité, la compétition et la territorialité, à la baisse dans un milieu homogène ou à la hausse dans un milieu hétérogène. La présence et le comportement des autres poissons tendent à entraîner une baisse de la distance interindividuelle et une augmentation de la nage en groupe ou en banc. Le risque de prédation en principe faible en aquaculture engendre une baisse du niveau de vigilance, des vitesses et distances d'échappement, des vitesses d'enfouissement et des capacités de camouflage.

Ces différentes réponses comportementales altérées par la pression de domestication peuvent être regroupées et présentées selon deux axes : (i) les comportements alimentaires, (ii) les comportements natatoires.

\section{1 / Les comportements alimentaires}

\section{Alimentation : régime, rythme et compétition}

La distribution d'aliments inertes entraîne le plus souvent une perte de la reconnaissance des organismes proies. Sous l'effet de la domestication, le poisson n'apporte plus guère d'attention à ses proies naturelles et s'intéresse prioritairement à l'aliment inerte artificiel. C'est le cas du bar d'élevage réintroduit en élevage semi-intensif en marais maritime (Reymond 1989, Bégout Anras et al 2001) chez lequel les plus gros individus utilisent surtout l'aliment tandis que les petits sont repoussés vers une recherche de proies naturelles.

Lactivité alimentaire spontanée est modulée par les facteurs biotiques, abiotiques et endogènes (Madrid et al 2001). Ainsi, en situation d'auto alimentation et en conditions expérimentales contrôlées, certaines espèces montrent des rythmes d'alimentation distincts : diurne (truite arc-en-ciel, Oncorhynchus mykiss), nocturne (atipa, Hoplosternum littorale) ou changeant avec la saison (bar, Dicentrarchus labrax), (Madrid et al 2001). Or, le contexte aquacole impose des périodes d'alimentation le plus souvent fixées par les contraintes d'élevage et peu liées à la chronobiologie, et des quantités distribuées auxquelles les poissons répondent à travers notamment l'activité locomotrice (changement de niveau, de rythme ou de distribution spatiale, Sanchez-Vazquez et Madrid
2001) qui peut avoir des conséquences marquées sur les potentiels de croissance et de conversion de l'aliment observés en cages aquacoles chez le saumon Atlantique (Salmo salar), le bar et la dorade (Sparus aurata) (Andrew et al 2002). D'autres réponses sont observées à travers notamment le déclenchement du comportement de demande alimentaire par action sur un automate (revue : Sanchez-Vazquez et Madrid 2001).

Une augmentation du nombre de compétiteurs modifie aussi les comportements alimentaires. Ainsi, à nourriture constante, l'investissement dans la tactique de " compétition en mêlée » qui implique une forte agitation dans la zone de nourrissage (scramble competition) diminue lorsque le nombre de compétiteurs augmente (Shaw et al 1995).

\section{2 / Les comportements natatoires}

\section{Distance interindividuelle}

Les poissons, comme les oiseaux, ne sont pas des animaux de contact et ils passent l'essentiel de leur temps à maintenir entre eux une distance plus ou moins importante, le plus souvent spécifique et compatible avec l'efficacité de l'activité en cours : c'est le territoire individuel de l'espèce. Des phénomènes d'adaptation sont observés en milieu confiné. En particulier, la distance interindividuelle dépend de l'origine des poissons, de la densité, du mode d'alimentation et des conditions environnementales (Tsukamoto et Uchida 1990). Ainsi chez l'ayu, Plecoglossus altivelis, les poissons étant soumis aux mêmes conditions expérimentales, les individus provenant du milieu sauvage maintiennent une certaine distance entre eux alors que ceux issus du milieu d'élevage perdent cette aptitude et se regroupent au contact les uns des autres (Tsukamoto et Uchida 1990). La distance interindividuelle résulte aussi d'un compromis entre compétition et prédation (Ruzzante 1994)

\section{Agressivité et comportement territorial}

Beaucoup de poissons défendent une certaine partie de leur territoire contre leurs semblables, ce territoire peut être le lieu de chasse, le nid. Le territoire peut être la propriété d'un individu, d'un couple ou d'un groupe d'individus, avec pour résultante une défense agressive de celui-ci. En conditions naturelles, la sélection de l'agressivité se fait alors sur le compromis entre le bénéfice énergétique de la nourriture acquise et protégée (ou augmentation de la survie de l'espèce dans le cas du nid) et l'énergie dépensée à la défense du territoire. L'un des objectifs majeurs de la domestication en ce qui concerne les poissons est sans nul doute la sélection de lignées d'individus non agressifs et à fort potentiel de croissance. Non agressivité et forte croissance sont assez souvent associées, la perte d'agressivité faisant réaliser aux individus de fortes économies d'énergie. Dans les élevages, la suppression d'agressivité ou le développement des individus à carac- 
tère neutre peut être liée à différentes conditions de l'environnement (Kestemont et Baras 2001). En règle générale, on tend à penser que l'environnement artificiel du milieu d'élevage réduit l'agressivité chez bon nombre de salmonidés (Petersson et Jarvi 2000). Mais ceci n'est qu'une apparence fortement liée aux caractéristiques des pratiques d'élevage. Les conditions environnementales et en particulier le caractère homogène ou hétérogène du système d'élevage pour les conditions abiotiques (hydrauliques, hydrologiques) et biotiques (accessibilité à l'aliment dans le temps et l'espace, densité et diversité de taille du cheptel) influencent tout à la fois l'agressivité, la territorialité et la compétition. Cela peut aboutir à favoriser les individus dominants au détriment des neutres et des dominés avec pour conséquence une amplification de l'hétérogénéité des tailles. L'occupation de l'espace dépend alors principalement de la taille des individus, de la présence des autres poissons, du niveau de faim et des conditions hydrauliques, hydrologiques et d'éclairement. Des choix d'habitat au sein de l'élevage en cage sont par exemple observés à travers des mouvements verticaux du saumon Atlantique selon les conditions thermiques et lumineuses (Juell et al 2003).

Concrètement, la mise en élevage peut inhiber le comportement territorial agressif, ce qui est souvent obtenu par de fortes densités : cas du tilapia du Nil (Oreochromis niloticus niloticus, Mélard 1986), de l'omble arctique (Salvelinus alpinus, Jorgensen et al 1993), du silure américain (Clarias gariepinus, Kaiser et al 1995, Hecht et Uys 1997) et du silure vundu (Heterobranchus longifile, Baras et al 1998). Des éclairements faibles ont le même effet chez l'omble arctique (Jørgensen et Jobling 1993), le silure vundu (Baras et al 1998) et le saumon coho (Oncorhynchus kisutch, Azuma et Iwata 1994) ; ou encore des courants modérés sont efficaces chez l'omble arctique (Jobling et al 1993).

\section{2 / Organes sensoriels et capacités comportementales modifiés au cours du développement en élevage}

De nombreux auteurs ont fait le constat d'un affaiblissement des performances sensorielles chez des poissons issus du milieu d'élevage, lié à la suppression de certaines contraintes et aux conditions de développement des animaux (Blaxter 1970, Howell 1994). Non seulement les seuils de sensibilité, les performances et les capacités comportementales (abaissement des seuils de vigilance) sont altérés, mais les structures anatomiques peuvent également être modifiées au cours du développement ontogénique.

Kawamura et al (1989) mettent en évidence un ralentissement important du développement des organes sensoriels (yeux, ligne latérale et odorat) chez de nombreuses larves en élevage. Des modifications anatomiques des organes sensoriels peuvent aboutir, comme chez le turbot (Psetta maxima), à un développement surnuméraire de branches de la ligne latérale (Ellis et al 1997a). Chez certaines espèces (salmonidés, ayu), des modifications des systèmes natatoires induisant une baisse de la capacité de saut et d'endurance ont été observées (Tsukamoto et Uchida 1990). Enfin, des modifications de certains comportements (par exemple nage en banc) peuvent être provoquées par l'alimentation via les apports d'acide ascorbique ou d'acide docosahexaenoique (DHA) (Sakakura et al 1998, Tsukamoto et al 1999).

L'abaissement du seuil de vigilance est lié à la non confrontation permanente à des prédateurs potentiels. La modification du seuil de vigilance est généralement repérée par un raccourcissement de la distance de fuite. Elle a pour conséquence d'accroître le potentiel métabolique du poisson et de réduire les mouvements de panique. En élevage, ces derniers sont souvent associés à une brusque détection d'une présence humaine ou à une activité humaine inhabituelle. La réduction de la distance de fuite est signalée chez bon nombre d'espèces comme différents Salmonidés (Olla et Davis 1989, Olla et al 1994), chez la morue (Gadus morhua, Nordeide et Svåsand 1990), et d'autres (Johnsson et al 2001). Dans ce domaine, la domestication de la truite arc-en-ciel a si bien réussi que toute activité près de son bassin a pour effet de stimuler sa demande alimentaire (Speare et al 1995). Cet abaissement du seuil de vigilance peut aussi être révélé par la disparition plus ou moins complète du tilting behaviour. Dans ce type de comportement, le poisson s'incline sur le côté en écartant les nageoires et en renforçant les contrastes de sa coloration, il traduit ainsi sont état d'alarme. On observe ceci chez la dorade japonaise Pagrus major (Uchida et al 1993).

La perte des comportements migratoires est aussi fréquemment observée chez les poissons d'élevage qui tendent soit à se sédentariser lorsqu'ils sont relâchés en milieu naturel (cas de truites arc-en-ciel triploïdes, Bridger et al 2001), soit à effectuer des déplacements à contre sens (cas des dévalaisons des ayus d'élevage au lieu d'une migration vers l'amont, Tsukamoto et al 1990). Cependant, comme observé chez les morues (Svåsand 1990), la domestication peut ne pas affecter ce comportement chez d'autres espèces.

L'adaptation chromatique et l'enfouissement sont des comportements surtout exprimés par les poissons plats. Le maintien en élevage a généralement pour conséquences un ralentissement de l'adaptation chromatique associé à un ralentissement du comportement fouisseur chez la sole Solea solea (Ellis et al 1997b) ou à la perte de celui-ci comme chez la plie japonaise Paralichthys olivaceus (Yamashita et Yamada 1999).

\section{Conclusions}

Dans le contexte des réflexions menées sur le repeuplement, Tsukamoto et al (1999) ont 
mis en avant l'importance de la qualité comportementale des juvéniles relâchés dans le milieu naturel. Ainsi ils ont définis plusieurs niveaux à intégrer pour assurer l'efficacité du repeuplement : une première sphère de santé qui inclut la physiologie et la morphologie des individus, puis une sphère de qualité qui englobe la sphère précédente en y ajoutant le comportement des individus afin d'optimiser la compatibilité avec l'écologie et le cycle de vie de l'espèce, sa survie et sa croissance. Parmi les critères de qualité comportementale, on trouve des indicateurs liés aux capacités migratoires (comportement de saut, de plongée, grégarisme, distance interindividuelle), aux capacités sensorielles (camouflage, vigilance) et aux capacités à s'alimenter en milieu naturel. Cette démarche analytique de la qualité comportementale peut être transposée aux élevages à visée aquacole. Ainsi, dans le cadre d'une typologie fonctionnelle des espèces piscicoles, il peut être formulé que les poissons plutôt territoriaux montrent des altérations des comportements liés à l'alimentation et à la compétition, alors que les poissons vivant en groupe ou en banc présentent plutôt des modifications des comportements natatoires et sociaux. Lidentification et la définition d'indicateurs comportementaux pertinents dans ce contexte aquacole, tels que le comportement alimentaire, l'agressivité, la distance interindividuelle et l'occupation de l'espace, permettront de mesurer de façon pratique la progression des processus de domestication dont certains traits seront recherchés en aquaculture alors qu'ils seront exclus en repeuplement. Toutefois ces indicateurs dépendent fortement du système et des conditions d'élevage, en particulier de la densité d'élevage. Ces mêmes indicateurs comportementaux pourront aussi servir à révéler des sélections génétiques ciblées sur certains caractères de croissance ou d'adaptabilité des poissons (Vandeputte et Prunet 2002).

\section{Références}

Andrew J.E., Noble C., Kadri S., Jewell H., Huntingford F.A., 2002. The effect of demand feeding on swimming speed and feeding responses in Atlantic salmon Salmo salar L., gilthead sea bream Sparus aurata L. and European sea bass Dicentrarchus labrax L. in sea cages. Aquac. Res., 33, 501-507.

Azuma T., Iwata M., 1994. Influences of illumination intensity on the nearest neighbour distance in coho salmon Oncorhynchus kisutch. J. Fish Biol., 45, 1113-1118.

Baras E., Tissier F., Westerloppe L., Mélard C., Philippart J.C., 1998. Feeding in darkness alleviates density-dependent growth of juvenile vundu catfish Heterobranchus longifilis (Claridae). Aquat. Living Resour.,11, 334-340.

Bégout Anras M.-L., Beauchaud M., Juell J.-E., Covès D. Lagardère J.-P., 2001. Environmental factors and feed intake: Rearing systems. In: Houlihan D., Boujard T., Jobling M. (eds), Food Intake in Fish, 157-188. Blackwell Science, Oxford, UK.

Blaxter J.H.S., 1970. Sensory deprivation and sensory input in rearing experiments. Helgoland. Mar. Res., 20, 642654 .

Boice R., 1972. Some behavioral tests of domestication in Norway rats. Behaviour, 42, 198-231.

Bridger C.J., Booth R.K., McKinley R.S., Scruton D.A. 2001. Site fidelity and dispersal patterns of domestic triploid steelhead trout (Oncorhynchus mykiss Walbaum) released to the wild. ICES J. Mar. Sci., 58, 510-516.

Eibl-Eibesfeldt I., 1972. Ethologie : biologie du comportement. R.G. Busnel (ed.), Editions Scientifiques NEB (Naturalia et Biologia), Jouy-en-Josas, France, 576 p.

Ellis T., Howell B.R., Hayes J., 1997a. Morphological differences between wild and hatchery-reared turbot. J. Fish Biol., 50, 1124-1128.

Ellis T., Howell B.R., Hughes R.N., 1997b. The cryptic responses of hatchery-reared sole to a natural sand substratum. J. Fish Biol., 51, 389-401.

Hale E.B. 1969. Domestication and the evolution of behaviour. In Hafez E.S.E. (ed), The behviour of domestic animals, 22-42. Bailliere, Tindall \& Cassell, London.

Hecht T., Uys W., 1997. Effect of density on the feeding and aggressive behaviour in juvenile African catfish, Clarias gariepinus. S. Afr. J. Sci., 93, 537-541

Howell B.R., 1994. Fitness of hatchery-reared fish for survival in the sea. Aquac. Fish. Manag., 25, 3-17.

Jobling M., Jorgensen E.H., Christiansen J.S., 1993 Growth performance of salmonids exposed to different flow regimes. In: Reinertsen H., Dahle L.A., Jorgensen L. Tvinnereim K. (eds), Fish Farming Technology, 301-305. Balkema, Rotterdam.

Johnsson J.I., Hojesjo J., Fleming I.A., 2001. Behavioural and heart rate responses to predation risk in wild and domesticated Atlantic salmon. Can. J. Fish. Aquat. Sci., 58 788-794.

Jorgensen E.H., Jobling M., 1993. Feeding in darkness eliminates density-dependent growth suppression in Arctic charr. Aquac. Int., 1, 90-93.

Jorgensen E.H., Christiansen J.S., Jobling M., 1993. Effects of stocking density on food intake, growth performance and oxygen consumption in artic charr (Salvenius alpinus). Aquaculture, 110, 191-204.

Juell J.E., Oppedal F., Boxaspen K., Taranger G.L., 2003. Submerged light increases swimming depth and reduces fish density of Atlantic salmon Salmo salar L. in productions cages. Aquac. Res., 34, 469-477.

Kaiser H., Weyl O., Hecht T., 1995. The effect of stocking density on growth, survival and agonistic behaviour of African catfish. Aquacult. Int., 3, 217-225.

Kawamura G., Mori H., Kuwahara A., 1989. Comparison of sense organ development in wild and reared flounde Paralichthys olivaceus larvae. Nippon Suisan Gakkaishi, $55,2079-2083$

Kestemont P, Baras E, 2001 Environmental factors and feed intake: Mechanisms and interactions. In: Houlihan D. Boujard T., Jobling M. (eds), Food Intake in Fish, 131-156. Blackwell Science, Oxford, UK.

Kohane M..J., Parson P.A., 1988. Domestication Evolutionary change under stress. Evol. Biol., 23, 31-48.

Madrid J.A., Boujard T., Sanchez-Vazquez F.J., 2001 Feeding Rhythms. In: Houlihan D., Boujard T., Jobling M. (eds), Food Intake in Fish, 189-215. Blackwell Science, Oxford, UK.

Mayr E., 1963. Animal species and evolution. Harvard Univeristy Press, Cambridge, MA, USA, 811 .

Mélard C., 1986. Les bases biologiques de l'élevage intensif du Tilapia du Nil. Cah. Ethol. Appl., 6, 242 p.

Nordeide J.T., Svasand T., 1990 The behaviour of wild and reared juvenile cod, Gadus morhua L., towards a potential predator. Aquac. Fish. Manag., 21, 317-325.

Olla B.L., Davis M.W., 1989. The role of learning and stress in predator avoidance of hatchery-reared coho salmon (Oncorhynchus kisutch) juveniles. Aquaculture, 76, 209-214. 
Olla B.L., Davis M.W., Ryer C.H., 1994. Behavioural deficits in hatchery-reared fish: potential effects on survival following release. Aquac. Fish. Manag., 25, 19-34.

Petersson E., Jarvi T., 2000. Both contest and scramble competition affect the growth performance of brown trout, Salmo trutta, parr of wild and sea-ranched origin. Env. Biol. Fish., 59, 211-218.

Price E.O., 1984. Behavioural aspects of animal domestication. Q. Rev. Biol., 59, 1-32.

Reymond H., 1989. Régime alimentaire du bar Dicentrarchus labrax en première et en deuxième année d'élevage semi-intensif en marais maritime : contribution relative des proies naturelles et de l'aliment à l'ingéré journalier. Rapport final, contrat SEMDAC 89-0001 et contrat IFREMER 88.5.524009.

Ruzzante D.E., 1994. Domestication effects on aggressive and schooling behavior in fish. Aquaculture, 120, 1-24.

Sakakura Y., Koshio S., Iida Y., Tsukamoto K., Kida T., Blom J.H., 1998. Dietary vitamin C improves the quality of yellowtail (Seriola quinqueradiata) seedlings. Aquaculture, 161, 425-434.

Sanchez-Vazquez F.J., Madrid J.A., 2001. Feeding Anticipatory Activity. In: Houlihan D., Boujard T., Jobling M. (eds), Food Intake in Fish, 216-232. Blackwell Science, Oxford, UK.

Shaw J.J., Tregenza T., Parker G.A., Harvey I.F., 1995. Evolutionary stable foraging speeds in feeding scrambles: a model and an experimental test. Proc. R. Soc. Lond. B, 260, 273-277.

Speare D.J., MacNair N., Hammell K.L., 1995: Demonstration of tank effect on growth indices of juvenile rainbow trout (Oncorhynchus mykiss) during an ad libitum feeding trial.. Am. J. Vet. Res., 56, 1372-1379.

Svåsand T., 1990. Comparisons of migration patterns of wild and recaptured coastal cod, Gadus morhua L., released in a small fjord in western Norway. Aquac. Fish. Manag., 21, 491-495.

Tsukamoto K., Uchida K., 1990. Spacing and jumping behaviour of the Ayu Plecoglossus altivelis. Nippon Suisan Gakkaishi, 56,1383-1392.

Tsukamoto K., Masuda S., Endo M., Otake T., 1990. Behavioural characteristics of the Ayu, Plecoglossus altivelis, as predictive indices for stocking effectiveness in rivers. Nippon Suisan Gakkaishi, 56, 1177-1186.

Tsukamoto K., Kuwada H., Uchida K., Masuda R., Sakakura Y., 1999. Fish quality and stocking effectiveness: Behavioural approach. In: Howell B.R., Moksness E. Svasand T. (eds), Stock enhancement and sea ranching, 205-218. Oxford, Malden, MA, USA.

Uchida K., Kuwada H., Tsukamoto K., 1993. Tilting behaviour, a fear response to frightening stimuli, as a possible predictive index for stocking effectiveness in the juveniles of red sea bream Pagrus major. Nippon Suisan Gakkaishi, 59, 991-999.

Vandeputte M., Prunet P., 2002. Génétique et adaptation chez les poissons : domestication, résistance au stress et adaptation aux conditions du milieu. INRA Prod. Anim., 15, 365-371.

Yamashita Y., Yamada H., 1999. Release strategy for Japanese flounder fry in stock enhancement programmes. In: Howell B.R., Moksness E., Svåsand T. (eds), Stock enhancement and sea ranching, 191-204. Oxford, Malden, MA, USA.

\section{Domestication and behaviour in fish.}

One of the major objective of domestication is the selection of lines with low aggressiveness and a high growth potential. Analyses of domesticated animals traits often illustrate numerous changes in behaviour due to rearing environment. However there are few reports of these changes in fish. In the present paper, we first describe how behaviour is affected by rearing and then discuss how sensory organs and behavioural abilities are modified during development under breeding conditions.
\end{abstract}

Abstract

Rearing conditions modify feeding behaviour particularly because food distribution do not necessary follow biological rhythm and food is often available in larger amount compared to wild-type situations. In particular food distribution mode may favour aggressiveness. Rearing conditions (stream, fish density...) are also susceptible to modify spatial distribution of fish and relationship between fish in their closed environment. In particular, fish often reduce their inter-indivi- dual distances which may also have detrimental influences on their aggressiveness.

Several behavioural abilities seem to be affected by development in breeding conditions and this appears to be related to modification of sensory organs. This includes modification of the lateral line system, eye and smell. These modifications are often associated to a lowering of watchfulness threshold which in turn modifies behaviour and particularly escape responses from potential predators. A modification of chromatic adaptation has also been shown for flat fishes.

In conclusion, the analysis of behavioural modifications is of utmost importance to identify indicators of domestication progress which must be taken into account to guarantee cultured fish welfare and thus rearing sustainability.

BEGOUT ANRAS M.L., LAGARDERE J.P., 2004 Domestication et comportement chez les poissons téléostéens. INRA Prod. Anim., 17, 211-215. 
